\title{
A STUDY ON LIVER FUNCTION TEST PROFILE OF 2ND LINE DRUGS IN MULTI DRUG RESISTANT (MDR) AND EXTENSIVELY DRUG RESISTANT (XDR) TUBERCULOSIS CASES REGISTERED UNDER DR-TB CENTRE IN A TERTIARY CARE HOSPITAL
}

\begin{tabular}{ll}
$\begin{array}{l}\text { Dr Rupam Kumar } \\
\text { Ta }\end{array}$ & $\begin{array}{l}\text { Associate Professor. Department of Pulmonary Medicine. Burdwan Medical } \\
\text { College, Burdwan }\end{array}$ \\
\hline Dr. Pronoy Sen * & $\begin{array}{l}2^{\text {rd }} \text { year Junior Resident, Department of Pulmonary Medicine. Burdwan } \\
\text { Medical College, Burdwan. }{ }^{*} \text { Corresponding Author }\end{array}$ \\
\hline
\end{tabular}

ABSTRACT BACKGROUND: The emergence of drug resistant mycobacterium has become a significant public health problem creating an obstacle to effective Tuberculosis (TB) control. Freedom from TB is possible with timely, regular, complete treatment, with assurance, prevention and management of side effects of antitubercular drugs. Present study was conducted to evaluate common and rare adverse drug reactions (ADR) of CAT IV and CAT V to analyse demographic, radiological and bacteriological profile and treatment outcome in MDR \&XDR patients.

Aims and Objectives- To evaluate the common and rare adverse drug reactions of intensive phase treatment of Multi Drug Resistant Tuberculosis (MDR) and Extensively Drug Resistant Tuberculosis (XDR) as per WHO-UMC Causality Assessment Scale.

METHODS: 76 patients of MDR and XDR Tuberculosis were admitted in DR-TB (Drug Resistant TB) centre, Burdwan Medical College and Hospital and the adverse drug reaction profile of 2 nd line drugs were analysed during the intensive phase for $a$ year after fulfilling the inclusion and exclusion criteria. Treatment was given as per guidelines by Revised National TB Control Program PMDT (Programmatic management of drug-resistant TB).

RESULTS: Adverse drug reactions on GI system were nausea 73 patients (96.10\%), vomiting 70 (92.10\%), acidity 41 (53.9\%), and sulphurous belching and hepatitis $1(1.31 \%)$ each.

CONCLUSIONS: The reactions were common in first 60 days of the regimen and in patients with BMI $\leq 18$. Hence vigilant monitoring is required for these types of patients during the initial period and sputum smear and culture conversion is very well correlated with clinical and radiological improvement.

KEYWORDS : Mdr Tb, Xdr Tb, Adverse Effects.

\section{BACKGROUND}

Mycobacterium tuberculosis (M. tuberculosis) is an ancient human pathogen, which has plagued countless human societies despite the introduction of curative and preventive therapy in the last century. Extensively drug-resistant TB (XDR TB) was first reported in 2006 but has now been documented on six continents ${ }^{1}$. These trends are critically important for global health, since drug-resistant TB mortality rates are high and second and third-line agents for the treatment of drugresistant TB are less potent and less tolerable than first-line therapies.

Drug resistance in tuberculosis is a global problem and India is no exception to this This study was taken up to determine the adverse drug reactions profile of a patient, previous history of anti tubercular drug intake and pattern of drug resistant. Globally, $5 \%$ of TB cases were estimated to have had MDR-TB in 2013 (3.5\% of new and $20.5 \%$ of previously treated TB cases). Drug resistance surveillance data show that an estimated 480,000 people developed MDR-TB in 2013 and 210,000 people died. Extensively drug-resistant TB (XDR-TB) has been reported by 100 countries in 2013 . On average, an estimated $9 \%$ of people with MDR-TB have XDR-TB 2 .

In India, the prevalence of multi-drug resistant TB (MDR-TB), defined as resistance to Isoniazid and Rifampicin with or without resistance to other drugs, is found to be at a low level in most of the regions. Data from several studies conducted by TRC and NTI, have found MDR-TB levels of less than $1 \%$ to $3 \%$ in new cases and around $12 \%$ in re-treatment cases ${ }^{3,4}$

India may be considered as one of the global epicentre of TB including the drug resistant one and many patients are being treated with second line anti-TB drugs. However, there is limited data of adverse drug reactions from the second line anti-TB drugs on the Indian patients. Indian patients are different from their global counterparts both by genetic structure and phenotype; hence prone to differ in anti TB drug action and pharmacokinetics also. Therefore, there is need for more data from the Indian patients related to second line antiTB drugs including the adverse drug reaction. Hence, the present study was been planned to systemically generate and analyze the adverse drug reaction data of the second line anti TB drugs on Eastern Indian patients.

\section{METHODS}

This cross sectional study was designed to include all patients receiving treatment for Drug Resistant TB over a period of one year. So there was no prespecified sample size for this study. This study was conducted at DR-TB centre, Burdwan Medical College and Hospital. Permission of Institutional Ethics Committee was obtained for the study. Written informed consents were obtained from all participating patients. 75 patients were included in the study. They were followed every month for 6 (9) months in intensive phase after the start of $2^{\text {nd }}$ line drugs and adverse drug reactions were recorded, as the maximum adverse reactions usually occur in this period. Treatment was given as per guidelines by Revised National TB Control Program PMDT (Programmatic management of drugresistant TB) (Erstwhile DOTS Plus). ${ }^{5}$

\section{INCLUSION CRITERIA}

1. Patient of age $>18$ years.

2. Both sexes.

3. Patients with proved drug resistant tuberculosis.

\section{EXCLUSION CRITERIA}

1. Patient admitted $<7$ days in DR-TB Centre.

\section{PARAMETERS STUDIED INCLUDE:}

1. HB\%, TC, DC, ESR

2. Blood urea, serum creatinine

3. Liver function test

4. FBS/PPBS

5. Sputum for AFB stain and gram stain

6. Line probe assay 
7. Urine albumin, sugar and microscopy

8. Chest X-RAY PA view.

After selection of each patient on the basis of inclusion and exclusion criteria a written informed consent was taken. Data was collected using a pretested pro forma meeting the objectives of the study. Detailed history, physical examination and necessary investigations were undertaken. The purpose of the study was explained to the patient and informed consent obtained.

At the end of the study the data was complied, tabulated for analysis.

All the collected data were analysed by using SPSS version 16 statistical software. Descriptive statistics were applied to the data. All data were presented as number and percent. Chi Square Test and Fisher exact test were applied wherever applicable to find out statistical differences and $p$ value $<0.05$ were considered statistically significant.

\section{RESULTS}

In our study among the 76 cases, most of them were pulmonary Tuberculosis (96\%) whereas only $4 \%$ were Extra Pulmonary Tuberculosis. Majority were from age group 21-30 (44\%), that is, the most productive age group of life. Majority $64 \%$ either studied upto $5^{\text {th }}$ standard or are illiterate (Fig l). Majority (95\%) live in a Kuchcha house. $32 \%$ of patients were farmers, $23 \%$ were housewives and $12 \%$ were labourers. Most of them (84.2\%) had no co morbidities but among the rest a significant number of patients (6.6\%) had Diabetes Mellitus. Majority 67 out of 76 had a history of incomplete ATD intake (88.2\%). Biological specimen: sputum CBNAAT/ LPA/ DST MTB detected, Rif resistant was found in 71 patients, MTB detected, Rif resistant by CBNAAT in FNAC of lymph node for 3 patients and both sputum \& pleural fluid CBNAAT MTB detected, Rif Resistant for 2 patients. Chest X-Ray features showed that a majority: 41 patients had bilateral consolidation and 21 patients had cavitary lesion. Overall compliance in IP: 63 patients had taken regular medication and 13 patients took irregular treatment or were lost to follow up. 3 patients died in each, regular and irregular treatment.

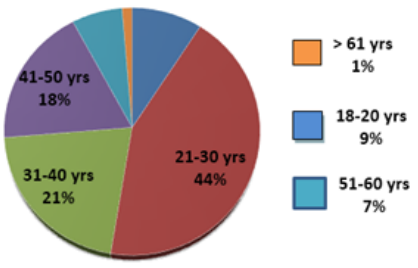

Figure 1. Distribution of Age Group in MDR and XDR TB Patients
Adverse drug reactions related to Gastro intestinal system like nausea, vomiting, heart burn and sulphurous belching were noted in most of the patients and these were more common after initiation of treatment (Fig 2). Among the GI related symptoms, incidences of emesis (nqusea \& vomiting) were higher than even cumulative of all other GI related ADRs and the difference was statistically significant $(p,<0.0001)$.

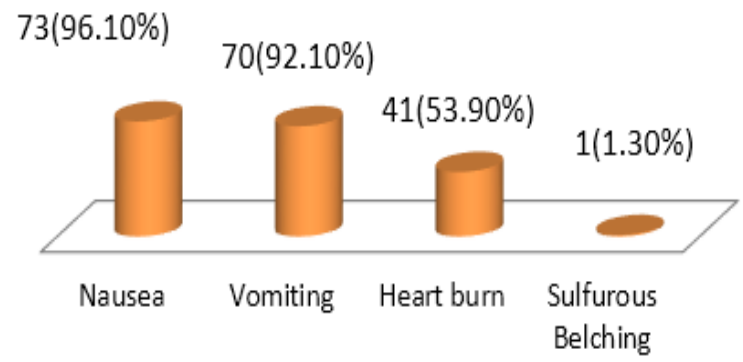

Figure 2: Gastrointestinal System related ADRs

Table 3: Elevation of Liver Enzymes

\begin{tabular}{|l|l|l|l|}
\hline Characteristic & No (n) & Percentage (\%) \\
\hline \multirow{2}{*}{ Serum ALP } & Elevated & 43 & 56.5 \\
\cline { 2 - 4 } & Not elevated & 33 & 43.5 \\
\hline
\end{tabular}

Table 4: Elevation of ALP enzyme

\begin{tabular}{|l|l|l|l|}
\hline Characteristic & No (n) & Percentage (\%) \\
\hline \multirow{2}{*}{ Serum SGPT } & Elevated & 3 & 3.9 \\
\cline { 2 - 4 } & Not elevated & 73 & 96.1 \\
\hline Serum SGOT & Elevated & 17 & 22.4 \\
\cline { 2 - 4 } & Not elevated & 59 & 77.6 \\
\hline
\end{tabular}

\section{DISCUSSION}

The present study has found that the second line anti-TB drugs are prone to produce adverse drug reactions in almost every patient. There was clustering of Gastrointestinal ADRs in the initial phases of treatment. However, in the later phase many patients suffered from neurological ADR. There were also reports of involvement of eye, liver, kidney, heart or skin in a number of patients.

Most of the patients in the present study had suffered from Gastro Intestinal related ADRs. This is corroborated with the other studies like Sangeta V et $\mathrm{al}^{6}$, Rohan Hire et $\mathrm{al}^{7}$, Dela $\mathrm{Ai}^{8}$.

Most of the patients had rise in serum ALP whereas only 3.9\% had rise in serum SGPT and $22 \%$ had raised SGOT.

Table 1. Comparison of Various Studies Related to ADRs of $2^{\text {nd }}$ Line Anti Tubercular Drugs

\begin{tabular}{|c|c|c|c|c|}
\hline Names of ADRs & $\begin{array}{c}\text { Our } \\
\text { Study }(\mathbf{n}=\mathbf{7 6})(\%)\end{array}$ & $\begin{array}{c}\text { Sangeta V et al } \\
\text { (Baroda, } \mathbf{n}=142) 6 \%\end{array}$ & $\begin{array}{c}\text { Rohan Hire et al } \\
\text { (Central India, } \mathbf{n}=110)^{7} \%\end{array}$ & $\begin{array}{c}\text { Dela } \mathrm{AI} \\
(\text { Gujarat, India } \mathbf{n}=\mathbf{7 2}) \mathbf{8} \%\end{array}$ \\
\hline GI related ADRs & 96 & 100 & 30 & 24.5 \\
\hline
\end{tabular}

Drugs were stopped or withdrawn in a number of patients. There are several possible explanations for the differences in the number of patients requiring drugs to be removed from the regimen due to $\mathrm{ADRs}$. These include genetic and phenotypic differences of the patients of Eastern India as well as variation in ability of the health care workers to detect ADRs and provide management.

The major strength of the study was complete follow up of the patients for a long duration. The study also utilized the standard tools like WHO-Uppsala Monitoring Center tool for causality assessment which is simple and widely used worldwide. However, there were few weaknesses in the study.
These include limited sample size, no formal sample size preestimation and possibility of under-reporting of ADRs. As the patients were assessed periodically, and reports of the symptoms were mostly dependent on the capacity of the patients to recall the ADRs, there remained the chances of recall bias and underreporting of non-serious ADRs.

\section{CONCLUSIONS}

Adverse Drug Reactions are common findings with second line anti TB drugs. Almost all major systems are affected by the ADRs due to these drugs though the large proportion is nonserious and self limiting. Gastrointestinal ADRs usually cluster around the initiation of treatment Patients with low BMI 
are more prone to develop ADRs. However, there is need for further studies to explore the serious ADRs and validation of the present findings in larger sample population.

\section{REFERENCES}

1. Report WHO/HTM/TB/2006.375 (World Health Organization, Geneva, 2006),Centers for Disease Control and Prevention. Revised definition of extensively drug-resistant tuberculosis. MMWR 2006; 55:1176)

2. World Health Organization. Multidrug and extensively drug-resistant TB(M/XDR-TB): 2014.

3. Mahadev B, Kumar P, Agarwal SP, Chauhan LS, Srikantaramu N. Surveillance of drug resistance to anti-tuberculosis drugs in districts of Hoogli in West Bengal and Mayurbhanj in Orissa. [Last cited on 2019 Dec 6]; Indian J Tuberc. 2005 52:5-10. Available from: http://www.imsear.li.mahidol.ac.th /handle/123 $456789 / 146942$.

4. Paramasivan CN, Venkataraman P, Chandrasekaran V, Bhat S, Narayanan PR. Surveillance of drug resistance in tuberculosis in two districts of South India. Int J Tuberc Lung Dis 2002: 6 (6); 479-84

5. Guidelines on Programmatic Management of Drug Resistant TB (PMDT) in India 2012 https://tbcindia.gov.in/WriteReadData/1892s/8320929355 Guid elines\%20for\%20PMDT\%20in\%20India\%20-\%20May\%202012.pdf

6. Patel S, Bhikhubhai N, Patel A, Shringarpure K, Mehta K, Shukla L, et al. Adverse Drug Reactions in Patients put on Multi Drug Resistant Tuberculosis (MDR-TB) Treatment in Seven Districts of Central Gujarat. Jour Young Pharmacists [Internet]. 2015:7(4); 425-31. [cited 2019 Dec 6] Available from: https://www.jyoungpharm.org/sites/default/files/10.5530jyp.2015.4s.3.pdf

7. Hire R, Kale AS, Dakhale GN, Gaikwad N. A prospective, observational study of adverse reactions to drug regimen for multi-drug resistant pulmonary tuberculosis in central India. Mediterr J Hematol Infect Dis. 2014;6(1): e2014061.

8. Dela AI, Tank NKD, Singh AP, Piparva KG. Adverse drug reactions and treatment outcome analysis of DOTS-plus therapy of MDR-TB patients at district tuberculosis centre: A four year retrospective study. Lung India. 2017 Dec;34(6):522-6.

9. Nathanson E, Gupta R, Huamani P, Leimane V, Pasechnikov AD, Tupasi TE, et al. Adverse events in the treatment of multidrug-resistant tuberculosis: results from the DOTS-Plus initiative. Int J Tuberc Lung Dis. 2004 Nov;8(11):1382-4. 